\title{
Generation of transgenic fibroblasts expressing pancreas-specific and doxycycline-inducible ICER I $\gamma$ for the establishment of a porcine model of human diabetes mellitus
}

\author{
HEE YOUNG KANG ${ }^{1}$, EUI-MAN JUNG ${ }^{1}$, EUI-JU HONG ${ }^{1}$, SANG-HWAN HYUN ${ }^{2}$, \\ WOO-SUK HWANG ${ }^{3}$ and EUI-BAE JEUNG ${ }^{1}$
}

${ }^{1}$ Laboratory of Veterinary Biochemistry and Molecular Biology; ${ }^{2}$ Laboratory of Veterinary Embryology and Biotechnology,
College of Veterinary Medicine, Chungbuk National University, Cheongju, Chungbuk 361-763;
${ }^{3}$ SooAm Biotech Research Foundation, Seoul 137-851, Republic of Korea

Received September 23, 2013; Accepted April 25, 2014

DOI: $10.3892 / \mathrm{mmr} .2014 .2255$

\begin{abstract}
Inducible cyclic AMP (cAMP) early repressor (ICER) I $\gamma$ acts as an endogenous inhibitor and disrupts the transcriptional regulation of cAMP response element binding protein (CREBP) responsive genes. Since the overexpression of ICER $I \gamma$ induces severe diabetes in a transgenic mouse model, with characteristics similar to human diabetes mellitus, an ICER I $\gamma$ construct containing an adjustable pancreas tissue specific promoter was utilized in the present study. Using the human insulin promoter region, a doxycycline (dox)-inducible ICER I $\gamma$ expression system was established using the tetracycline (tet)-controlled transactivator (tTA) with a TA response element (TRE) promoter. A unitary tet-on system that combined a tet-on activator cassette was also developed and was controlled by the human insulin promoter with a responder cassette containing genes encoding ICER I $\gamma$ regulated by the TRE promoter. To determine whether doxenhanced ICER I $\gamma$ expression affected insulin production, the unitary tet-on ICER I $\gamma$ vector was introduced into a mouse pancreatic $\beta$-cell line and then the cells were treated with $0.1-1 \mathrm{mg} / \mathrm{ml}$ dox. The results revealed a robust increase in ICER I $\gamma$ expression and decreased insulin production. Therefore, this in vitro system may be useful for studying human diabetes mellitus and pre-diabetes using tissue-specific promoters and a dox-inducible transgene. In addition, porcine transgenic fibroblasts containing dox-inducible ICER I $\gamma$ were generated. These fibroblasts may serve as a cell source for
\end{abstract}

Correspondence to: Dr Eui-Bae Jeung, Laboratory of Veterinary Biochemistry and Molecular Biology, College of Veterinary Medicine, Chungbuk National University, 52 Naesudong-ro, Heungduk-gu, Cheongju, Chungbuk 361-763, Republic of Korea E-mail: ebjeung@chungbuk.ac.kr

Key words: pig, fibroblast, diabetes mellitus, inducible cyclic AMP early repressor I $\gamma$, dox-inducible ICER I $\gamma$, human insulin promoter somatic cell nuclear transfer to generate a porcine model of human diabetes mellitus.

\section{Introduction}

The expression of insulin genes in pancreatic islet cells is affected by plasma glucose or intracellular cAMP $(1,2)$. cAMP response element binding protein (CREBP) stimulates the transcription of insulin genes by binding to CREs in the promoter region $(3,4)$. Among the CREB family members, CRE modulator (CREM) is a unique gene that encodes either transcriptional activators or repressors based on alternative splicing (5). In particular, inducible cAMP early repressor (ICER) has an important role as a dominant-negative regulator of CREB and CREM activity, and consists of DNA binding and leucine zipper domains, while also lacking an $\mathrm{N}$-terminal transactivation domain. As a strong repressor of cAMP-induced gene expression, the ICER acts as an endogenous inhibitor competing with CREB on the CRE sequence, and then ICER I $\gamma$ induces the return of cAMP signaling to the basal state (6). Of note, prolonged expression of ICER I $\gamma$ results in the development of pathological conditions. Previously, it was reported that the overexpression of ICER I $\gamma$ is associated with an increased incidence of diabetes and decreased $\beta$-cell numbers in transgenic mice $(7,8)$.

When using constructs with a constitutively active promoter, the unregulated expression of a target gene often produces unexpected results. To avoid these limitations, a conditional transgenic technique was adopted, the tetracycline (tet)-on system. This system enables both temporal and spatial regulation of transgene expression, and requires a responder construct and activator construct in a single cell (9). Transcriptional activator protein (tTA; a transactivator) consists of a modified bacterial tet repressor (TetR) fused to three minimal activation domains of the herpes simplex virus transcription activator (VP16). Expression of this construct is controlled by tetracycline or its analog, doxycycline (dox) (10). In the presence of dox, a conformational change in tTA allows the activator to bind to the tTA-response element (TRE) promoter of a responder construct, and induces transcription 
Table I. Primer sequences.

\begin{tabular}{|c|c|c|c|}
\hline Primer name & Restriction enzyme & Direction & Sequences $\left(5^{\prime}\right.$ to $\left.3^{\prime}\right)$ \\
\hline Human insulin promoter $(-1,432)$ & SpeI & Forward & ACT AGT TAC CCC AGG GGC TCA GCC CAG ATG \\
\hline Human insulin promoter $(+1)$ & EcoRI & Reverse & GAA TTC GGC CAG CAG CGC CAG CAG G \\
\hline ICER I $\gamma$ cDNA & MluI & Forward & ACG CGT ATG GCT GTA ACT GGA GAT GA \\
\hline ICER I $\gamma$ cDNA & BamHI & Reverse & GGA TCC CTA ATC TGT TTT AGG AGA GCA AAT G \\
\hline Tet-response cassette & Bst1107I & Forward & GTA TAC CGA GGC CCT TTC GTC TTC AAG AAT TC \\
\hline Tet-response cassette & SpeI & Reverse & ACT AGT GCC GCA GAC ATG ATA AGA TAC ATT GA \\
\hline Confirming primer a & & Forward & GTG CTG ACG ACC AAG GAG AT \\
\hline Confirming primer a' & & Reverse & TTT CAG AAG TGG GGG CAT AG \\
\hline Confirming primer $\mathrm{b}$ & & Forward & GAG GAT GGA GCA GTT TGC AT \\
\hline Confirming primer $\mathrm{b}^{\prime}$ & & Reverse & GCA TTC CAC CAC TGC TCC CA \\
\hline ICER I $\gamma$ & & Forward & ATG GCT GTA ACT GGA GAT GA \\
\hline ICER I $\gamma$ & & Reverse & CTA ATC TGT TTT AGG AGA GCA AAT G \\
\hline Insulin-1 & & Forward & CCC TGT TGG TGC ACT TCC TA \\
\hline Insulin-1 & & Reverse & CAC TTG TGG GTC CTC CAC TT \\
\hline Mouse $\beta$-actin & & Forward & ACA GGC ATT GTG ATG GAC TC \\
\hline Mouse $\beta$-actin & & Reverse & ATT TCC CTC TCA GCT GTG GT \\
\hline
\end{tabular}

ICER I $\gamma$, inducible cyclic AMP early repressor I $\gamma$.

of the target gene and a green fluorescent protein reporter gene downstream of the TRE promoter. The TRE promoter consists of seven repeats of a 19-bp tet operator sequence located upstream of a minimal cytomegalovirus (CMV) promoter in which the binding sites for endogenous mammalian transcription factors have been eliminated (11).

In the present study, a pancreas-specific, dox-inducible ICER I $\gamma$ expression vector was constructed, where the CMV promoter was exchanged for the human insulin promoter in the activator construct. Additionally, this construct contained a unitary system combined with an activator cassette and responder cassette. Although in vivo experiments designed to study diabetes have been conducted in rodents and other animals $(12,13)$, the phenotypic hallmarks of diabetes mellitus in rodent models are not sufficient to fully elucidate the mechanisms underlying the development of diabetes in humans. Therefore, porcine transgenic fibroblasts were also established, that were genetically modified to evoke type 1 diabetes mellitus-like symptoms in a porcine model.

\section{Materials and methods}

Cell culture. The study was approved by the Ethics Committee of Chungbuk National University (Cheongju, Korea). The porcine fibroblasts were obtained from miniature pig fetuses (Yucatan pigs; Optifarm Solution Inc., Gyeonggi-do, Korea) on the thirtieth day of pregnancy and the cells were routinely maintained in Dulbecco's Modified Eagle's Medium (DMEM) containing $25 \mathrm{mM}$ glucose, supplemented with $10 \%$ fetal bovine serum (FBS; WelGENE, Inc., Daejeon, South Korea), $100 \mathrm{U} / \mathrm{ml}$ penicillin and $100 \mu \mathrm{g} / \mathrm{ml}$ streptomycin. The mouse $\beta$-cell line, MIN6 (American Type Culture Collection, Manassas, VA, USA), was cultured in DMEM containing $25 \mathrm{mM}$ glucose, supplemented with $15 \% \mathrm{FBS}, 55 \mu \mathrm{M}$ 2-mercaptoethanol (Gibco, Grand island, NY, USA), 100 U/ml penicillin and $100 \mu \mathrm{g} / \mathrm{ml}$ streptomycin. All cells were grown in a humidified $5 \% \mathrm{CO}_{2}$ atmosphere at $37^{\circ} \mathrm{C}$. Unless otherwise indicated, all cell culture materials were obtained from Invitrogen Life Technologies (Carlsbad, CA, USA).

Genomic DNA extraction and PCR. Genomic DNA from the cells was isolated with a G-DEX ${ }^{\mathrm{TM}}$ IIc Genomic DNA Extraction kit (iNtRON Biotechnology, Inc., Seoul, South Korea). Genomic DNA $(0.1 \mu \mathrm{g})$ was amplified in a $20-\mu 1$ PCR reaction containing $1 \mathrm{U}$ i-Start Taq polymerase (iNtRON Biotechnology, Inc.), 2 mM dNTPs (Takara Bio, Inc., Shiga, Japan) and 10 pmol of each specific primer. The details of all primers are described in Table I. The PCR reactions were denatured at $94^{\circ} \mathrm{C}$ for $30 \mathrm{sec}$, annealed at $62^{\circ} \mathrm{C}$ for $30 \mathrm{sec}$ and extended at $72^{\circ} \mathrm{C}$ for $1-2 \mathrm{~min}$. The PCR products were subjected to cloning processes and/or separated on a $1 \%$ agarose gel, stained with ethidium bromide and photographed under UV illumination. The image was scanned using GelDoc EQ (Bio-Rad, Hercules, CA, USA).

Vector construction. All restriction enzymes were obtained from Takara Bio, Inc. The human insulin promoter region (from nucleotides (nt) $-1,431$ to $+1 \mathrm{nt} ;+1=$ the transcriptional start site) demonstrated the highest transcriptional activity in a previous study (14) and were prepared by long-range PCR using human genomic DNA (Clontech Laboratories, Mountain View, CA, USA) as the template, and specific primers containing restriction enzyme sites (SpeI at the $5^{\prime}$ end or EcoRI at the $3^{\prime}$ end). Amplified fragments were digested with SpeI and EcoRI and replaced with the $\mathrm{CMV}$ promoter of the transcriptional activator construct, pCMV-Tet3G, purchased from Clontech Laboratories. The ICER I $\gamma$ cDNA was prepared by PCR using genomic DNA from pig pancreas as the template and was 
inserted into pTRE3G-ZsGreen1 which contains green fluorescence protein, ZsGreen1 (Clontech Laboratories) through MluI at the 5' end or BamHI at the 3' end. Using the PCR method, the ICER I $\gamma$-expressing tTA-response region obtained from recombinant pTRE3G-ZsGreen1-pig ICER I $\gamma$ construct was digested with Bst1107I or SpeI and combined with the recombinant pHINSP-Tet3G vector controlled by the human insulin promoter.

Establishment of transgenic cell lines. The fibroblasts were transfected with the linearized targeting vector using Lipofectamine ${ }^{\circledR} 2000$ (Invitrogen, Carlsbad, CA, USA). Following $24 \mathrm{~h}$ of transfection, the medium was replaced with DMEM supplemented with $10 \%$ FBS and $250 \mu \mathrm{g} / \mathrm{ml} \mathrm{G}-418$ (Roche Diagnostics, Indianapolis, IN, USA) for four weeks. The antibiotic-resistant cells were further selected, subjected to PCR-based genotyping and stored until required for somatic cell nuclear transfer.

Transient transfection and dox treatment. Transient transfection was performed using Lipofectamine ${ }^{\mathrm{TM}} 2000$ according to the manufacturer's instructions. Briefly, $3 \times 10^{5}$ cells were seeded in 6-well tissue culture plates one day prior to transfection. In total, $4 \mu \mathrm{g}$ of the recombinant constructs per well was transfected into the cells under serum-free DMEM. Following incubation for $4 \mathrm{~h}$, the medium was replaced with DMEM containing $10 \%$ FBS, $100 \mathrm{U} / \mathrm{ml}$ penicillin and $100 \mu \mathrm{g} / \mathrm{ml}$ streptomycin. A total of $20 \mathrm{~h}$ later, various concentrations of dox were treated in transiently transfected cells for an additional $24 \mathrm{~h}$.

RNA preparation and semi-quantitative (q)PCR. Total RNA from MIN6 cells was extracted using TRIzol reagent (Invitrogen Life Technologies) according to the manufacturer's instructions. The concentration of the total RNA was determined by measuring the absorbance at $260 \mathrm{~nm}$. First-strand cDNA was prepared by subjecting total RNA $(1 \mu \mathrm{g})$ to reverse transcription using Moloney murine leukemia virus (MMLV) reverse transcriptase (Invitrogen Life Technologies) and random primers (9-mers; Takara Bio, Inc.). To determine the optimal conditions for logarithmic phase PCR amplification for target cDNA, aliquots of total cDNA $(1 \mu \mathrm{g})$ were amplified using different numbers of cycles. The mouse $\beta$-actin gene was amplified as the internal control to rule out the possibility of RNA degradation and to control for variations in mRNA concentrations. A linear correlation between PCR product band visibility and the number of amplification cycles was observed for the target mRNA. The mouse $\beta$-actin and target genes were quantified using 28 and 30 cycles, respectively. The PCR reactions were denatured at $94^{\circ} \mathrm{C}$ for $30 \mathrm{sec}$, annealed at $58^{\circ} \mathrm{C}$ for $30 \mathrm{sec}$ and extended at $72^{\circ} \mathrm{C}$ for $30 \mathrm{sec}$. The PCR products were on a $2.3 \%$ agarose gel, stained with ethidium bromide and photographed under UV illumination. The image was scanned using GelDoc EQ (Bio-Rad).

Western blot analysis. Lysates were prepared in RIPA buffer (50 mM Tris, $\mathrm{pH} 7.4,150 \mathrm{mM}$ sodium chloride $(\mathrm{NaCl})$, $1 \%$ Triton X-100, $0.5 \%$ sodium deoxycholate, $1 \mathrm{mM}$ EDTA and $1 \mathrm{mM}$ PMSF) and protease inhibitor cocktail (Roche Diagnostics). Protein content was determined using the Pierce
BCA Micro Protein Assay kit (Thermo Fisher Scientific, Waltham, MA, USA). Total proteins obtained by centrifugation $\left(13,000 \mathrm{x} \mathrm{g}\right.$ for $20 \mathrm{~min}$ at $\left.4^{\circ} \mathrm{C}\right)$ were denatured at $95^{\circ} \mathrm{C}$ for $5 \mathrm{~min}$. A total of $20-50 \mu \mathrm{g}$ of protein were size-separated by electrophoresis on $13 \%$ SDS-polyacrylamide gels and electrophoretically transferred to a nitrocellulose membrane. Non-specific binding was blocked with TBST $(20 \mathrm{mM}$ Tris, $\mathrm{pH} 7.6,137 \mathrm{mM} \mathrm{NaCl}$ and $0.05 \%$ Tween-20) containing 5\% non-fat milk for $2 \mathrm{~h}$ at room temperature (RT). The membrane was probed with the following primary antibodies for $2 \mathrm{~h}$ at RT: rabbit polyclonal antibody to Insulin (H-86, sc-9168), diluted 1:1,000; rabbit polyclonal antibody to CREM (X-12, sc-440), diluted 1:1,000 and rabbit polyclonal antibody to $\beta$-actin (N-21, sc-130656), diluted 1:1,000. All antibodies were purchased from Santa Cruz Biotechnology, Inc. (Santa Cruz, CA, USA). The membrane was subsequently exposed to a horseradish peroxidase-conjugated secondary antibody (1:5,000 dilution in TBST) for $1 \mathrm{~h}$ at RT.

\section{Results}

Establishment of a unitary tet-on ICER I $\gamma$ expression vector. The unitary tet-on system was composed of an activator cassette and responder cassette (Fig. 1A). The activator cassette had a tTA under the control of the human insulin promoter (-1,432 to $+1 \mathrm{nt})$ which is associated with maximum promoter activity in mouse MIN6 $\beta$-cells line according to our previous study (14). The responder cassette contained the porcine ICER I $\gamma$ target gene and cDNA encoding ZsGreen1 green fluorescence protein. The expression of both genes was controlled by a TRE promoter. tTA was specifically transcribed in pancreas cells under the control of the human insulin promoter, underwent a conformational change induced by the administration of dox and then bound to the TRE promoter of the responder cassette. Finally, the key gene downstream of the TRE promoter, ICER I $\gamma$, was specifically transcribed in the pancreas cells. Additionally, expression of the green fluorescence marker, ZsGreen1, was observed.

Generation and characterization of fibroblast cell lines containing the dox-inducible porcine ICER I $\gamma$ construct. The dox-inducible porcine ICER I $\gamma$ vector was linearized and used to transfect miniature pig fibroblasts using a liposomal-mediated gene delivery system. The transfected fibroblasts were selected and maintained with a medium containing G418 $(250 \mu \mathrm{g} / \mathrm{ml})$ for four weeks. Chromosomal integration of the targeting vector was confirmed by a PCR-based method using primer sets specific for the vector (Table I). Genomic DNA extracted from G418-resistant colonies was amplified with primers a and a' (product size, 992 bp; Fig. 1B) or primers b and b' (product size, 1,585 bp; Fig. 1C). In total, 12 positive colonies were obtained following two rounds of transfection (Table II). Fibroblasts from the positive colonies may serve as a cellular source for somatic cell nuclear transfer to generate a porcine model that overexpresses ICER I $\gamma$ in a dox-inducible, pancreas-specific manner.

Observation of dox-inducible fluorescence in MIN6 cells and porcine fibroblasts. To observe tissue-specific expression, we transiently transfected mouse pancreatic $\beta$-cells (MIN6) and porcine non-pancreatic fibroblasts. The cells were 
A
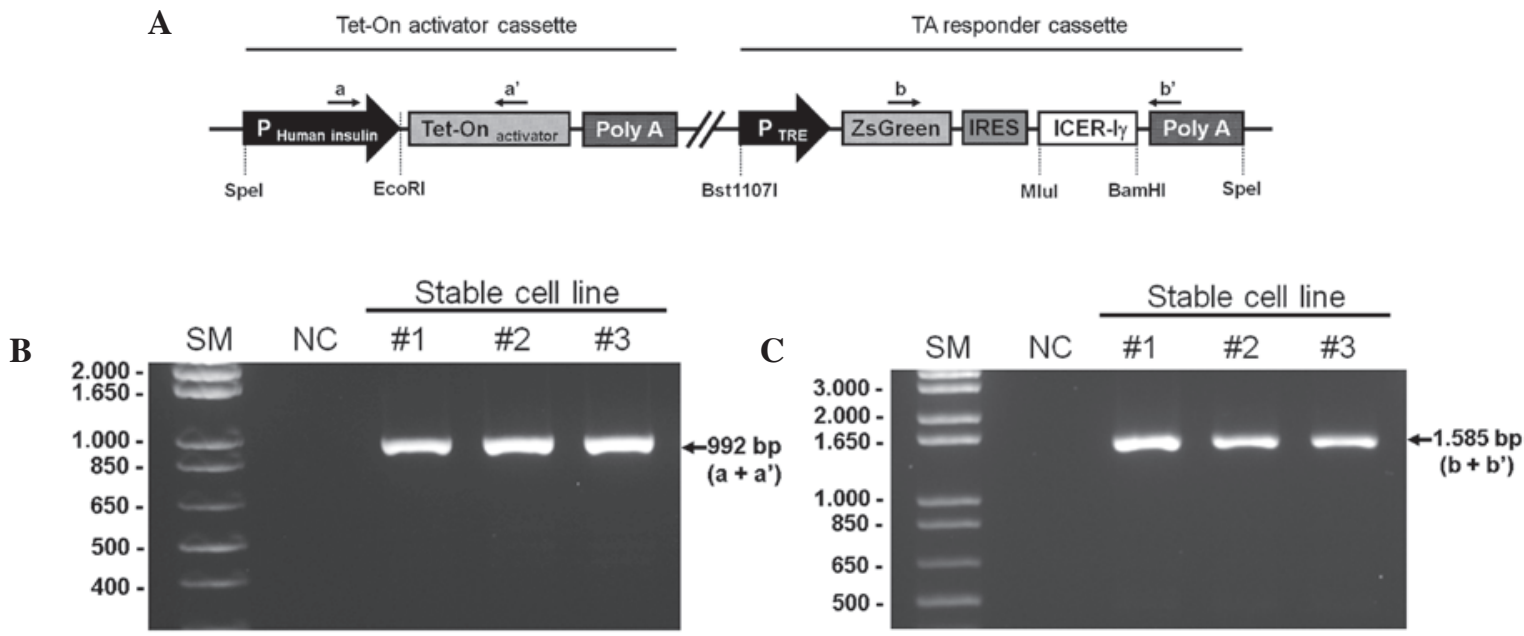

Figure 1. Schematic structure of the targeting vector and PCR-based confirmation of transgenic fibroblasts. (A) The unitary tet-on system was composed of two parts in a vector; an activator cassette and a responder cassette. The activator cassette had tTA under the control of the human insulin promoter (-1,432 to $+1 \mathrm{nt})$. The responder cassette contained the target gene, porcine ICER I $\gamma$ and ZsGreen1 cDNA expressing green fluorescence protein, both which are controlled by the TRE promoter. This unitary vector was linearized and integrated into the genomic DNA of the porcine fibroblasts. Genomic DNA was isolated from G418-resistant fibroblast colonies and was identified with specific primer sets indicated by the arrows (a, a', b, b'). (B) The PCR products with primer a and a' represents the chromosomal insertion of the activator cassette. (C) Whether the same fibroblast colonies had responder cassette was confirmed using primer b and b'. IRES, internal ribosomal entry site; SM, size marker; NC, negative control; ICER I $\gamma$, inducible cyclic AMP early repressor I $\gamma$; tTa, tet-controlled transactivator; TRE, tTA-response element.

A

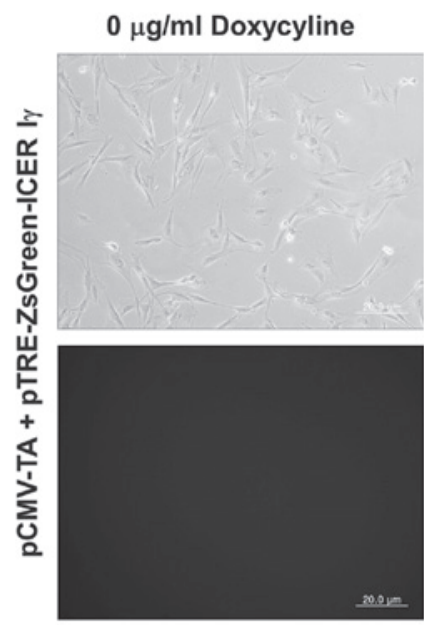

B

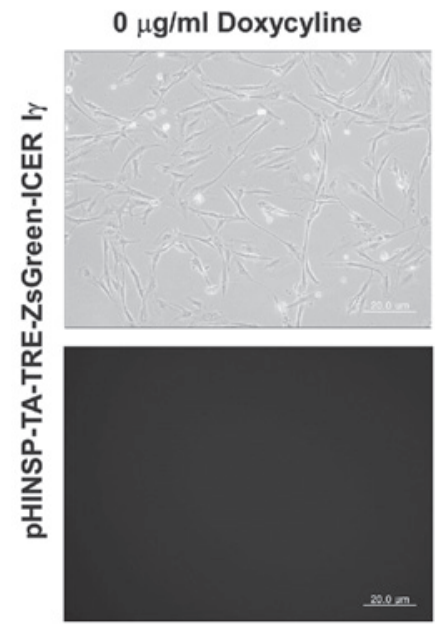

$0.1 \mu \mathrm{g} / \mathrm{ml}$ Doxycyline
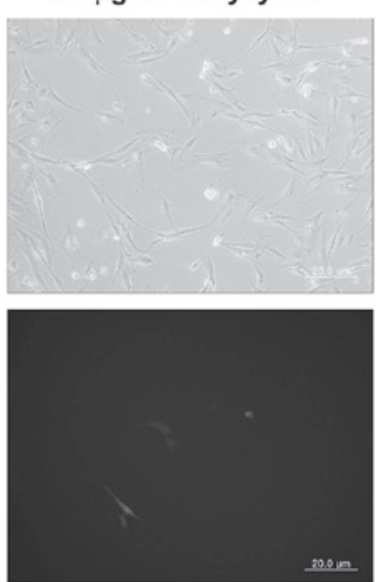

$0.1 \mu \mathrm{g} / \mathrm{ml}$ Doxycyline
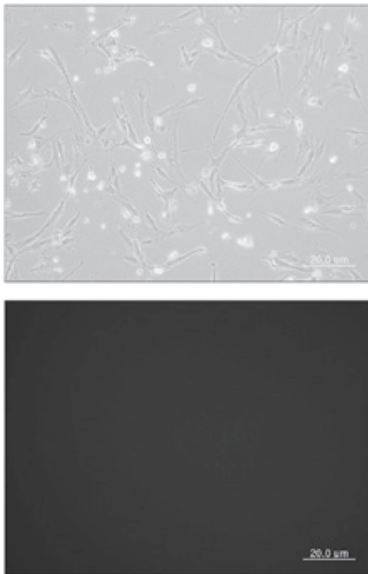

$1 \mu \mathrm{g} / \mathrm{ml}$ Doxycyline
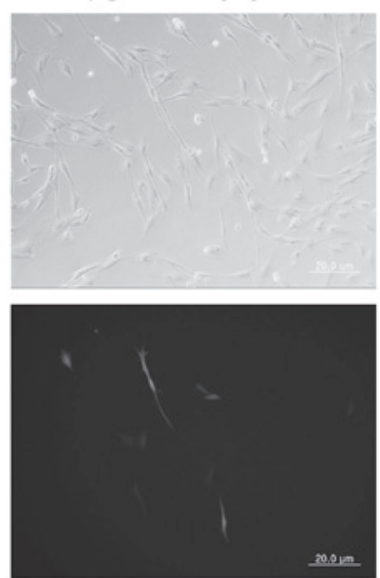

$1 \mu \mathrm{g} / \mathrm{ml}$ Doxycyline

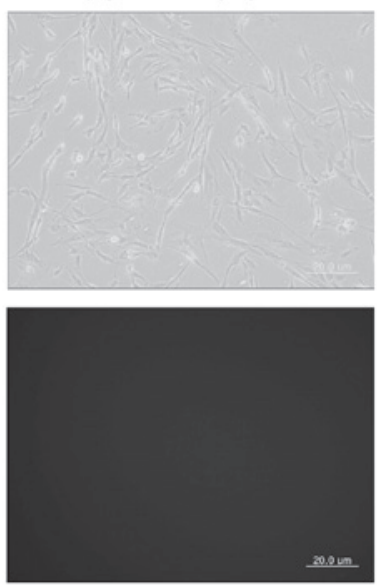

Figure 2. Observation of dox-inducible green fluorescence in mouse $\beta$-cell line, MIN6, by transient transfection of pCMV-TA with (A) pTRE-ZsGreenICER I $\gamma$ or (B) unitary pHINSP-TA-TRE-ZsGreen-ICER I $\gamma$. In the MIN6 cell line, dox-inducible and pancreas specific expression of green fluorescence by human insulin promoter was observed. Furthermore, the green fluorescence demonstrated dox-dose dependent expression, as treated dox concentration was increased to $0,0.1$ and $1 \mathrm{mg} / \mathrm{ml}$. CMV, cytomegalovirus promoter; TA, tet-on transcription activator; HINSP, human insulin promoter; ICER I $\gamma$, inducible cyclic AMP early repressor I $\gamma$; TRE, tTA-response element. 
A

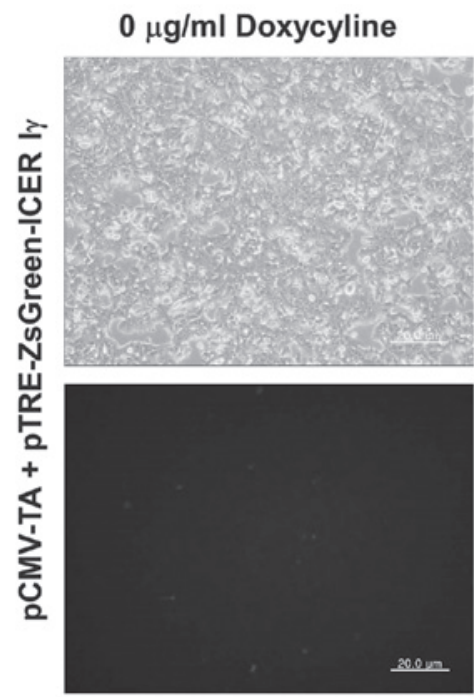

B

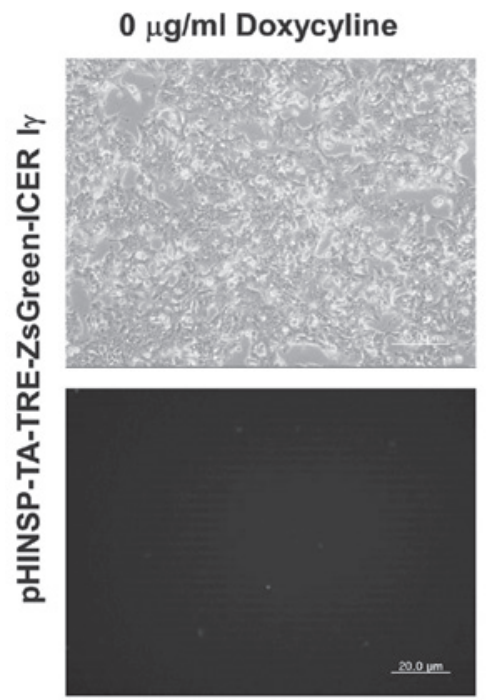

$0.1 \mu \mathrm{g} / \mathrm{ml}$ Doxycyline
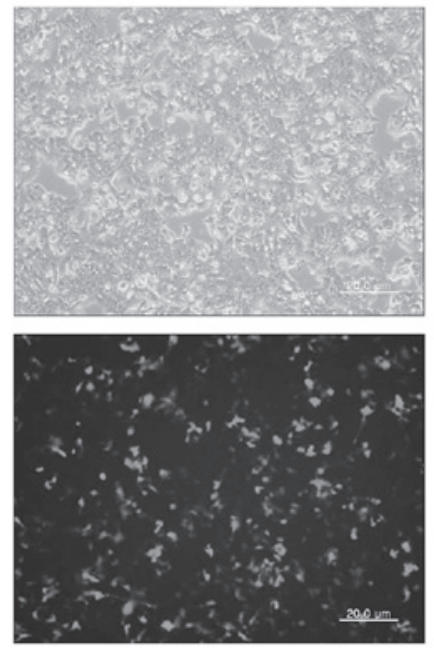

$0.1 \mu \mathrm{g} / \mathrm{ml}$ Doxycyline
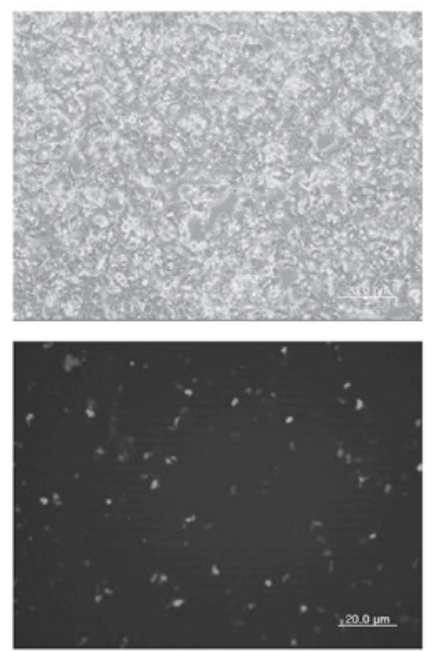

$1 \mu \mathrm{g} / \mathrm{ml}$ Doxycyline
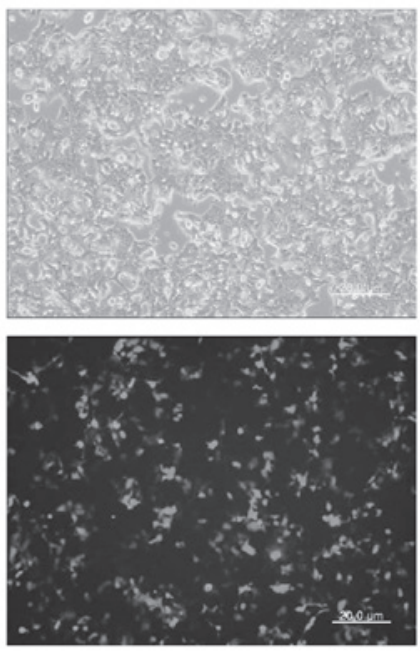

$1 \mu \mathrm{g} / \mathrm{ml}$ Doxycyline
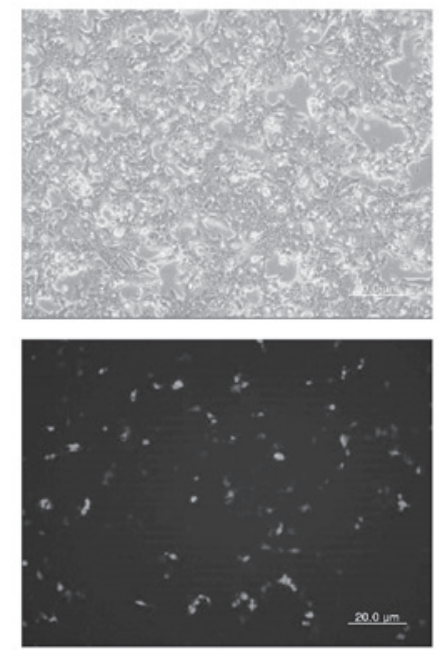

Figure 3. Observation of dox-inducible green fluorescence in porcine fibroblasts by transient transfection of pCMV-TA with (A) pTRE-ZsGreen-ICER I $\gamma$ or (B) unitary pHINSP-TA-TRE-ZsGreen-ICER I $\gamma$. Treated dox concentration was increased to $0,0.1 \mathrm{and} 1 \mathrm{mg} / \mathrm{ml}$. CMV, cytomegalovirus promoter; TA, tet-on transcription activator; HINSP, human insulin promoter; ICER I $\gamma$, inducible cyclic AMP early repressor I $\gamma$; TRE, tTA-response element; dox, doxycycline.

co-transfected with pCMV-TA and pTRE-ZsGreen1-ICER I $\gamma$ constructs as a positive control to confirm the activity of the dox-inducible expression system. In the MIN6 cells expressing pHINSP-TA-TRE-ZsGreen-ICER I $\gamma$, we observed green fluorescence indicative of dox-inducible and pancreas-specific ICER I $\gamma$ expression (Fig. 2). In this regard, green fluorescence was also confirmed following dox treatment in a dose-dependent manner $(0,0.1$ and $1 \mu \mathrm{g} / \mathrm{ml})$. However, no fluorescence was observed in either the untreated or dox-treated porcine fibroblasts transiently transfected with pHINSP-TA-TRE-ZsGreen-ICER I $\gamma$ due to the pancreas specific insulin promoter (Fig. 3).

Dox-inducible porcine ICER I $\gamma$ mRNA expression in mouse $\beta$-cells. To evaluate dox-inducible ICER I $\gamma$ expression under the control of the human insulin promoter, reverse transcription (RT)-PCR was performed using mRNA obtained from transiently transfected MIN6 cells and porcine fibroblasts. Consistent to the fluorescence microscopy findings, dox-dose dependent and pancreas specific mRNA expression of ICER I $\gamma$ was observed in MIN6 cells (Fig. 4A) following dox
Table II. Transfection efficiencies of the porcine fibroblasts.

\begin{tabular}{lcc}
\hline & \multicolumn{2}{c}{ Colony (n) } \\
\cline { 2 - 3 } Transfection trials (n) & G418 resistant & PCR positive \\
\hline 2 & 12 & 12 \\
\hline
\end{tabular}

treatment but not in porcine fibroblasts (data not presented). Additionally, the expression levels of ICER I $\gamma$ in cells transfected with only pCMV-TA or pTRE-ZsGreen1-ICER I $\gamma$ were unchanged regardless of the absence or presence of dox. Although ICER I $\gamma$ expression in MIN6 cells expressing the pTRE-ZsGreen1-ICER I $\gamma$ transgene was observed, the level was consistently low and unaffected regardless of the presence of dox. It should be considered that unknown factors of the transcriptional environment may regulate the gene expression of in vitro systems through introduction of the target gene. 
A
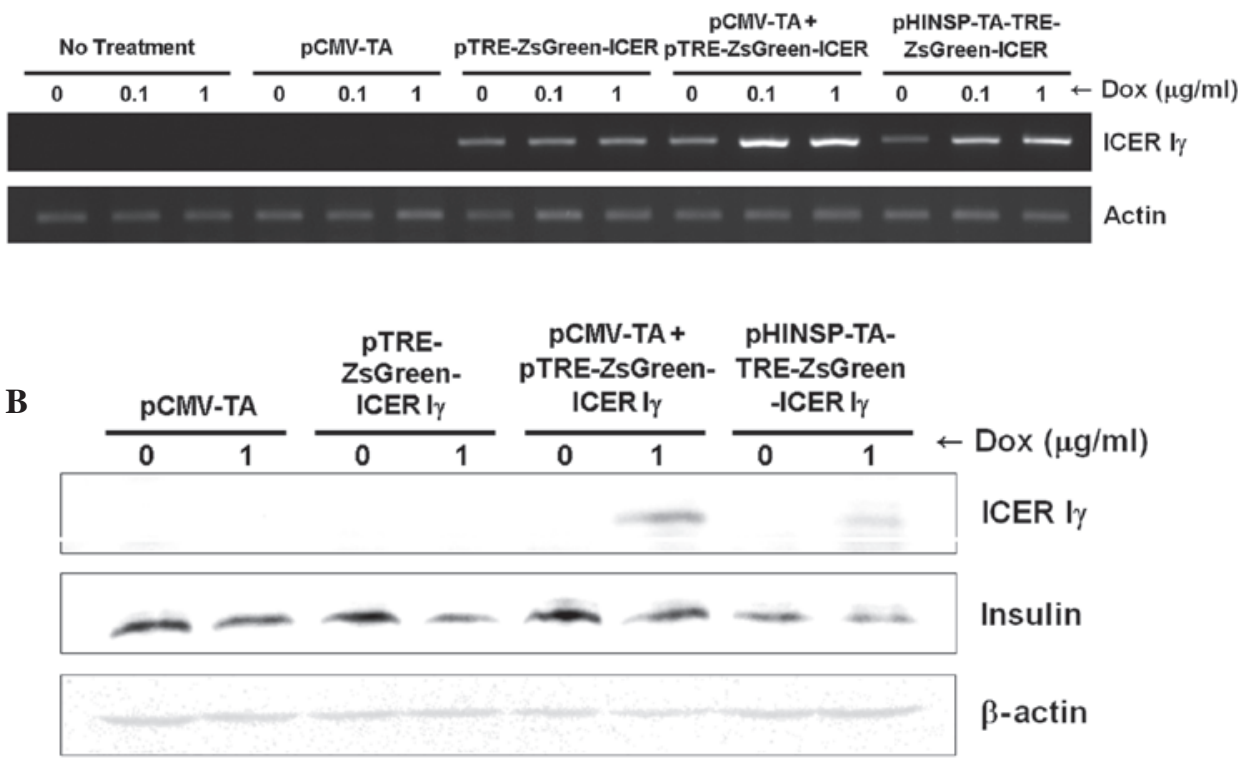

Figure 4. Dox-inducible porcine ICER I $\gamma$ mRNA and protein expression in the mouse $\beta$-cell line, MIN6. (A) Dox-dose dependent mRNA overexpression of ICER I $\gamma$ in both pCMV-TA with pTRE-ZsGreen-ICER I $\gamma$ and pHINSP-TA-TRE-ZsGreen-ICER I $\gamma$ transiently transfected MIN6 cells using RT-PCR. (B) ICER I $\gamma$-protein overexpression by dox administration revealed downregulation of insulin in MIN6 cells using western blot analysis. CMV, cytomegalovirus promoter; TA, tet-on transcription activator; HINSP, human insulin promoter; ICER I $\gamma$, inducible cyclic AMP early repressor I $\gamma$; TRE, tTA-response element; dox, doxycycline; RT-PCR, reverse transcription-PCR.

Insulin production in mouse $\beta$-cells expressing the dox-inducible porcine ICER I $\gamma$ gene. Since the ICER I $\gamma$ transgene represses insulin production in vivo $(7,8)$, western blot analysis was performed to determine whether dox-inducible expression of porcine ICER I $\gamma$ may inhibit insulin generation. The expression of insulin was evaluated in transiently transfecting MIN6 cells with four combinations of the constructs (Fig. 4B). ICER I $\gamma$ protein in cells transfected with only pCMV-TA or pTRE-ZsGreen1-ICER I $\gamma$ was not expressed regardless of the absence or presence of dox. Dox-inducible expression of ICER I $\gamma$ was observed in cells co-transfected with pCMV-TA and pTRE-ZsGreen1-ICER I $\gamma$, or transfected with pHINSP-TATRE-ZsGreen1-ICER I $\gamma$ alone. By contrast, insulin expression was decreased in the presence of dox. These findings indicated that dox-inducible ICER I $\gamma$ expression decreases insulin expression in mouse pancreas $\beta$-cell. Therefore, the extensive induction of ICER I $\gamma$ expression may interfere with insulin synthesis and promote the development of type 1 diabetes mellitus. As a result, our dox-inducible ICER I $\gamma$ expression system may be a useful tool for studying diabetes mellitus and pre-diabetes in humans by controlling ICER I $\gamma$ expression levels through dox administration.

\section{Discussion}

In rodent models, it has been reported that ICERs have an important role in T-cell mediated downregulated expression of interleukin (IL)-2, an essential growth factor for auto-aggressive T-effector cells $(15,16)$. In pancreatic $\beta$-cells, ICER I $\gamma$ suppresses not only insulin gene transcription but also cell replication via the reduction of cyclin A levels $(7,17)$. Therefore, the prolonged or constitutive expression of ICER results in the development of pathological conditions (7) similar to severe cases of diabetes mellitus, because ICER I $\gamma$ overexpression significantly blocks CRE-mediated transcription by competing with CRE-binding activators $(6,18)$. Therefore, in the present study, a tissue-specific ICER I $\gamma$ vector was constructed, whose expression was controlled by dox, and a porcine fibroblast cell line with this construct was also established to generate transgenic piglets.

The insulin promoter directly regulates insulin gene transcription according to plasma glucose levels and enables $\beta$-cells to produce insulin $(19,20)$. On this basis, the insulin promoter was utilized in the present study as a $\beta$-cell-specific regulator of gene transcription. The human insulin promoter region spanning $-1,431$ to +1 nt significantly increased ICER I $\gamma$ gene expression in MIN6 cells in the presence of high glucose levels and dox (Fig. 4A). However, induction of ICER I $\gamma$ expression in MIN6 cells slightly downregulated insulin production (Fig. 4B). It was hypothesized that the activity of the porcine ICER I $\gamma$ protein would not be maximized in mouse $\beta$-cells because the porcine ICER I $\gamma$ protein is documented as having 92.6-94.4\% identity to mouse ICERs, using a BLAST tool. By contrast, it may be possible to reduce the interaction between porcine ICER I $\gamma$ and mouse insulin promoter regulatory elements.

It was reported that the relative number and composition of principal insulin promoter regulatory elements in different species was determined through transcription factor binding site turnover and accretion (21-23). In particular, CRE is a key determinant of gene expression $(24,25)$ and binds to the widest array of transcription factors in the insulin promoter (26). Unlike the human insulin promoter that contains four copies of CREs, the mouse insulin promoter only has a single copy (23). For this reason, the binding rate of overexpressed ICER I $\gamma$ within the mouse insulin promoter may decrease and the repression of insulin expression by ICER I $\gamma$ may be weaker in spite of ICER I $\gamma$ overexpression via the human insulin promoter in mouse $\beta$-cells. 
MIN6 cells were derived from a transgenic C57BL/6 mouse insulinoma expressing an insulin-promoter/T-antigen construct and possess characteristics of pancreatic $\beta$-cells, including the ability to secrete insulin in response to glucose $(27,28)$. Although the amount of insulin secreted from MIN6 cells in the presence of a high $(25 \mathrm{mM})$ glucose concentration is six-to seven-fold greater than that observed with a low $(5 \mathrm{mM})$ glucose level, it has been noted that glucose-induced insulin secretion from the MIN6 cells may suddenly be lost during the course of passaging (28). This may result in a poor response to the downregulation of glucose-mediated insulin production via dox-inducible ICER I $\gamma$ expression.

To generate a porcine model for diabetes or metabolic syndromes, pig fibroblast cell lines expressing dox-inducible ICER I $\gamma$ were established. These cells should be a useful source for somatic cell nuclear transfer procedures. Porcine models provide various advantages for studying human metabolic syndromes because pigs are mono-gastric omnivores and have anatomical/physiological characteristics similar to that of humans (29). The porcine model established using the created cells may offer information crucial for understanding the mechanisms underlying human diabetes mellitus.

In the present study, the dox-inducible, tissue-specific expression of the transcriptional repressor ICER I $\gamma$ was observed in a mouse pancreatic $\beta$-cell line. This result was compared with the expression of ICER I $\gamma$ observed in transiently transfected primary porcine fibroblasts. The unitary tet-on ICER I $\gamma$ induction system was pancreas-specific and dox-inducible. When generating transgenic animals, these cellular traits may reduce stillbirth rates during pregnancy and unwanted outcomes caused by uncontrollable gene expression.

\section{Acknowledgments}

This study was supported by a grant from the Next-Generation BioGreen 21 Program (no. PJ00956301), Rural Development Administration, Republic of Korea.

\section{References}

1. Leonard J, Serup P, Gonzalez G, Edlund T and Montminy M: The LIM family transcription factor Isl-1 requires cAMP response element binding protein to promote somatostatin expression in pancreatic islet cells. Proc Natl Acad Sci USA 89: 6247-6251, 1992.

2. Barnett DW, Pressel DM, Chern HT, Scharp DW and Misler S: cAMP-enhancing agents 'permit' stimulus-secretion coupling in canine pancreatic islet beta-cells. J Membr Biol 138: 113-120, 1994.

3. Ortmeyer HK: Insulin decreases skeletal muscle cAMP-dependent protein kinase (PKA) activity in normal monkeys and increases PKA activity in insulin-resistant rhesus monkeys. J Basic Clin Physiol Pharmacol 8: 223-235, 1997.

4. Seino S, Takahashi H, Fujimoto W and Shibasaki T: Roles of cAMP signalling in insulin granule exocytosis. Diabetes Obes Metab 11 (Suppl 4): 180-188, 2009.

5. Foulkes NS, Borrelli E and Sassone-Corsi P: CREM gene: use of alternative DNA-binding domains generates multiple antagonists of cAMP-induced transcription. Cell 64: 739-749, 1991.

6. Mioduszewska B, Jaworski J and Kaczmarek L: Inducible cAMP early repressor (ICER) in the nervous system - a transcriptional regulator of neuronal plasticity and programmed cell death. J Neurochem 87: 1313-1320, 2003.

7. Inada A, Hamamoto Y, Tsuura Y, Miyazaki J, Toyokuni S, Ihara Y, Nagai K, Yamada Y, Bonner-Weir S and Seino Y: Overexpression of inducible cyclic AMP early repressor inhibits transactivation of genes and cell proliferation in pancreatic beta cells. Mol Cell Biol 24: 2831-2841, 2004.
8. Inada A, Someya Y, Yamada Y, Ihara Y, Kubota A, Ban N, Watanabe R, Tsuda K and Seino Y: The cyclic AMP response element modulator family regulates the insulin gene transcription by interacting with transcription factor IID. J Biol Chem 274: 21095-21103, 1999.

9. Gossen $\mathrm{M}$ and Bujard $\mathrm{H}$ : Tight control of gene expression in mammalian cells by tetracycline-responsive promoters. Proc Natl Acad Sci USA 89: 5547-5551, 1992.

10. Zhou X, Vink M, Klaver B, Berkhout B and Das AT: Optimization of the Tet-On system for regulated gene expression through viral evolution. Gene Ther 13: 1382-1390, 2006.

11. Gossen M, Freundlieb S, Bender G, Müller G, Hillen W and Bujard $\mathrm{H}$ : Transcriptional activation by tetracyclines in mammalian cells. Science 268: 1766-1769, 1995.

12. Saudek F: Gene therapy in the treatment of diabetes mellitus. Cas Lek Cesk 142: 523-527, 2003 (In Czech).

13. Driver JP, Serreze DV and Chen YG: Mouse models for the study of autoimmune type 1 diabetes: a NOD to similarities and differences to human disease. Semin Immunopathol 33: 67-87.

14. Jung EM, Kim YK, Lee GS, Hyun SH, Hwang WS and Jeung EB: Establishment of inducible cAMP early repressor transgenic fibroblasts in a porcine model of human type 1 diabetes mellitus. Mol Med Rep 6: 239-245.

15. Bodor J, Fehervari Z, Diamond B and Sakaguchi S: ICER/CREM-mediated transcriptional attenuation of IL-2 and its role in suppression by regulatory T cells. Eur J Immunol 37: 884-895, 2007.

16. Bodor J, Spetz AL, Strominger JL and Habener JF: cAMP inducibility of transcriptional repressor ICER in developing and mature human T lymphocytes. Proc Natl Acad Sci USA 93: 3536-3541, 1996.

17. Inada A, Weir GC and Bonner-Weir S: Induced ICER Igamma down-regulates cyclin A expression and cell proliferation in insulin-producing beta cells. Biochem Biophys Res Commun 329: 925-929, 2005.

18. Molina CA, Foulkes NS, Lalli E and Sassone-Corsi P: Inducibility and negative autoregulation of CREM: an alternative promoter directs the expression of ICER, an early response repressor. Cell 75: 875-886, 1993.

19. Odagiri H, Wang J and German MS: Function of the human insulin promoter in primary cultured islet cells. J Biol Chem 271: 1909-1915, 1996.

20. Burkhardt BR, Loiler SA, Anderson JA, Kilberg MS, Crawford JM, Flotte TR, Goudy KS, Ellis TM and Atkinson M: Glucose-responsive expression of the human insulin promoter in HepG2 human hepatoma cells. Ann NY Acad Sci 1005: 237-241, 2003.

21. Rockman MV and Wray GA: Abundant raw material for cisregulatory evolution in humans. Mol Biol Evol 19: 1991-2004, 2002.

22. Ludwig MZ and Kreitman M: Evolutionary dynamics of the enhancer region of even-skipped in Drosophila. Mol Biol Evol 12: 1002-1011, 1995.

23. Hay CW and Docherty K: Comparative analysis of insulin gene promoters: implications for diabetes research. Diabetes 55: 3201-3213, 2006.

24. Daniel PB, Walker WH and Habener JF: Cyclic AMP signaling and gene regulation. Annu Rev Nutr 18: 353-383, 1998.

25. Inagaki N, Maekawa T, Sudo T, Ishii S, Seino Y and Imura H: c-Jun represses the human insulin promoter activity that depends on multiple cAMP response elements. Proc Natl Acad Sci USA 89: 1045-1049, 1992.

26. Foulkes NS and Sassone-Corsi P: Transcription factors coupled to the cAMP-signalling pathway. Biochim Biophys Acta 1288: F101-F121, 1996.

27. Ishihara H, Asano T, Tsukuda K, Katagiri H, Inukai K, Anai M, Kikuchi M, Yazaki Y, Miyazaki JI and Oka Y: Pancreatic beta cell line MIN6 exhibits characteristics of glucose metabolism and glucose-stimulated insulin secretion similar to those of normal islets. Diabetologia 36: 1139-1145, 1993.

28. Miyazaki J, Araki K, Yamato E, Ikegami H, Asano T, Shibasaki Y, Oka Y and Yamamura K: Establishment of a pancreatic beta cell line that retains glucose-inducible insulin secretion: special reference to expression of glucose transporter isoforms. Endocrinology 127: 126-132, 1990.

29. Petersen B, Carnwath JW and Niemann H: The perspectives for porcine-to-human xenografts. Comp Immunol Microbiol Infect Dis 32: 91-105, 2009. 